\title{
Do Antineoplastic Drugs Play an Additional Role in the Progression of Non-Compaction Cardiomyopathy? A Case Report
}

\author{
Chaodi Luo, Yanjie Yang, Chun Yang, Xiang Hao, Zhenzhen Duan, Guoliang Li, Gang Tian* \\ Department of Cardiology, First Affiliated Hospital of Xi'an Jiaotong University, Xi'an, China \\ Email: *tiangang@xjtu.edu.cn
}

How to cite this paper: Luo, C.D., Yang, Y.J., Yang, C., Hao, X., Duan, Z.Z., Li, G.L. and Tian, G. (2020) Do Antineoplastic Drugs Play an Additional Role in the Progression of Non-Compaction Cardiomyopathy? A Case Report. Case Reports in Clinical Medicine, 9, 144-153.

https://doi.org/10.4236/crcm.2020.96021

Received: May 15, 2020

Accepted: June 2, 2020

Published: June 5, 2020

Copyright $\odot 2020$ by author(s) and Scientific Research Publishing Inc. This work is licensed under the Creative Commons Attribution International License (CC BY 4.0).

http://creativecommons.org/licenses/by/4.0/

\begin{abstract}
Non-compaction cardiomyopathy is a rare form of cardiomyopathy; its most common clinical manifestations are heart failure (HF), ventricular arrhythmia, thromboembolism, and sudden cardiac death. We report a rare case of a 63-year-old man with chest tightness, worsening lower leg edema, dyspnea, and decreased exercise tolerance. He had a medical history of gastric cancer treated with subtotal gastrectomy and post-operative chemotherapy with paclitaxel and fluorouracil three years ago. At that time, he was diagnosed with non-compaction cardiomyopathy, and the thickened and reticulated trabecular muscle was exclusively confined to left ventricular apex. Five months ago, he was admitted to our hospital with heart failure and treated for dilated cardiomyopathy, echocardiography revealed severe trabecular noncompact myocardium in both ventricles, which was confirmed by cardiac magnetic resonance imaging (CMR). It is generally accepted that non-compacted myocardium forms in the early embryonic stage, which raises a question in our case whether acquired factors, such as antineoplastic drugs, potentially accelerate the pathological progression of non-compaction cardiomyopathy. Considering there are disparities between current screening tools such as echocardiography and CMR regarding diagnostic criteria, multi-detector CT may be an alternative examination method that could provide a new perspective for diagnosis.
\end{abstract}

\section{Keywords}

Non-Compaction Cardiomyopathy, Antineoplastic Drugs, Heart Failure, Diagnosis

\section{Introduction}

Non-compaction cardiomyopathy is a congenital cardiomyopathy characterized 
by a distinctive ("spongy") morphological appearance of the ventricular myocardium. Non-compaction predominantly involves the distal (apical) portion of the left ventricle (LV) with deep intertrabecular recesses (sinusoids) in communication with the ventricular cavity, resulting from an arrest in normal embryogenesis [1]. Feldt et al. first described non-compaction of the ventricular myocardium on autopsy in 1969 [2]. The gross anatomical appearance is characterized by numerous, excessively prominent trabeculations and deep intertrabecular recesses [3]. Non-compaction cardiomyopathy can be familial. In one study, 6 of 34 patients (18\%) had a family history of left ventricular non-compaction (LVNC) [4]. The most common clinical manifestations are heart failure (HF), ventricular arrhythmia, thromboembolism, and sudden cardiac death. The condition is diagnosed by two-dimensional echocardiography or magnetic resonance imaging [5]. We report a rare case of a patient who was admitted to our hospital with clinical symptoms of HF and a history of gastric cancer treated with subtotal gastrectomy and post-operative chemotherapy with paclitaxel and fluorouracil. After further examination, he was diagnosed with biventricular non-compaction cardiomyopathy with biventricular thrombosis.

\section{Case Presentation}

A 63-year-old man was admitted to the hospital with chest tightness, worsening lower leg edema, dyspnea, and decreased exercise tolerance. The patient complained that he had these symptoms for 1 year and was getting worse, during which there was no regular treatment. He had no family history of sudden cardiac death, and he denied any history of drinking or smoking. The patient had gastric cancer treated with subtotal gastrectomy and a month-interval of post-operative chemotherapy with 4 cycles of paclitaxel (150 mg on day 1$)$ and fluorouracil (50 mg on day 1 - 14) 3 years ago. Echocardiography before the surgery suggested the left ventricular apical trabecular muscle became thickened and reticulated but cardiac function was normal, but he did not take it seriously and refused to do further cardiac examination and treatment. Five months ago, he was admitted to our hospital with dyspnea and abdominal distension. According to his medical records at our hospital, physical examination showed a remarkable systolic murmur of grade $4 / 6$ over the left second intercostal area without radiation to the carotids and a pansystolic murmur of grade $3 / 6$ at the apex without radiation, as well as jugular venous distention. The chest examination revealed decreased thorax movement, vocal tremor, and a decrease in breath sounds bilaterally. The 12-lead electrocardiogram showed sinus rhythm with 2 nd degree type 1 atrioventricular block. The echocardiogram revealed whole heart enlargement and systolic dysfunction characterized by a left ventricular ejection fraction (LVEF) as low as $26 \%$, but his sonographer did not notice the presence of trabeculae in the ventricular myocardium. Moreover, the results of the coronary computed tomographic angiography (CTA) were normal. Thus, the patient was initially diagnosed as dilated cardiomyopathy (DCM) and benefited from the treatment. 
Despite standard treatment for DCM, he was admitted again to our hospital with worsening symptoms of heart failure (HF). The echocardiogram revealed that an enlarged heart with mitral and aortic valve insufficiency, severe systolic dysfunction characterized by an left ventricular ejection fraction (LVEF) as low as $23 \%$, restrictive diastolic dysfunction, including peak $\mathrm{E}$ (early diastolic)/A (late diastolic) velocity $>1.5$, shortened deceleration time of the E-wave, and slightly hyperechoic masses in the left and right ventricular chambers, the biggest of which measured $2.52 \times 2.33 \mathrm{~cm}$ (Figure $1(\mathrm{a})$ ). The 12-lead electrocardiogram showed non-persistent ventricular tachycardia and 2nd degree type 1 atrioventricular block. The D-dimer and tumor biomarkers were normal. The patient's medical history, symptoms, and examination (especially the presence of biventricular mobile masses) were strongly suggestive of thrombus. As the patient was willing to undergo repeated blood tests for anticoagulant treatment and undertake bleeding risks, we tentatively used warfarin at alternate dosages of $1.875 \mathrm{mg} /$ day and $1.25 \mathrm{mg} /$ day, aiming for an international normalized ratio (INR) level of 2.0 - 3.0. Echocardiographic reexaminations 2 days later showed that the volume of the masses in the left and right ventricular cavities decreased significantly, which confirmed that the mobile masses were thrombi (Figure 1 (b) and Figure 1(c)). Considering the thrombus could mobilize at any time, we continued to use warfarin for anticoagulation, administered diuretics, and administered the calcium sensitizer, levosimendan at dosage of $12.5 \mathrm{mg}$, to improve cardiac function. After 6 days of comprehensive treatment, the patient's condition improved significantly.

To further clarify the thrombus situation, echocardiography was performed again, the results of which revealed that the area of left ventricular thrombus significantly decreased and the right ventricular thrombus disappeared. Furthermore, we found a severely thin myocardial wall and the appearance of prominent trabeculations in the left ventricle (Figure 1(d)). Two-dimensional CMR in the axial and sagittal planes showed highly trabeculated non-compacted myocardium involving the apical and lateral segments and a thin compact epicardial layer with massive thrombus formation between the trabeculae; the ratio of the thickness of the non-compact to compact myocardium was 3.7, more than 2.3 (Figure 2(a) and Figure 2(b)). According to the results of the echocardiography and CMR, the patient was diagnosed with non-compaction cardiomyopathy. Since there are no proposed guidelines for the management of patients with LVNC and the patient have evidence of severe LV dysfunction, the therapeutic treatment for this patient was basically limited to pharmacotherapy for heart failure and thrombosis.

In view of the above results, we believed that the patient received a clinical misdiagnosis five months ago. Moreover, we found that a large amount of non-compacted myocardium were present in the ventricular wall, mainly in the left ventricular wall, and a large number of thrombus were present in the ventricular cavity when we conducted multiplanar reformation based on the results of the coronary CTA performed five months ago (Figure 3(a) and Figure 3(b)). 
Non-compaction cardiomyopathy is a genetically heterogenous disorder, with both X-linked as well as autosomal dominant inheritance. Because of the potential genetic influence, we performed a series of examinations for the patient's 38and 31-year-old daughters; neither showed signs of heart failure and the electrocardiogram was normal. In order to clarify the situation of their hearts, transthoracic echocardiography was performed, the results of both revealed a thickened and reticulated left ventricular apical trabecular muscle (Figure 4(a) and Figure 4(b)). Sadly, the two daughters refused further CMR.

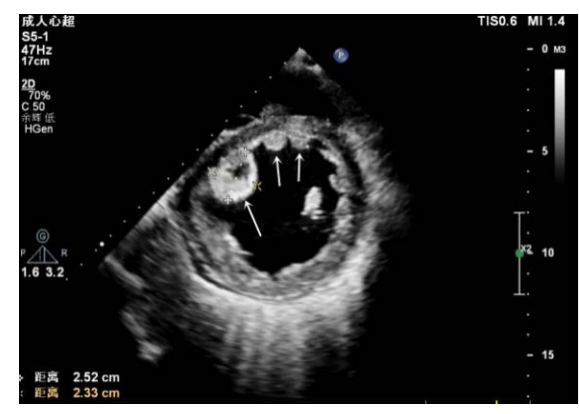

(a)

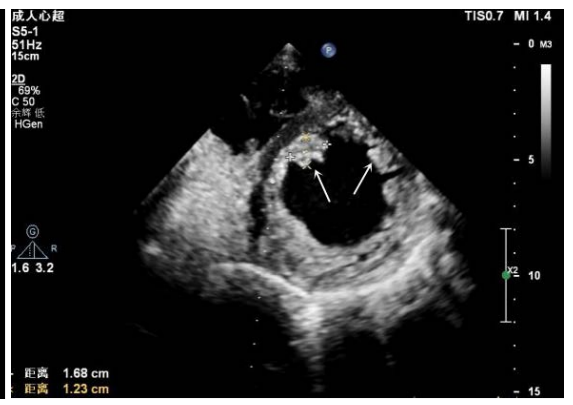

(b)

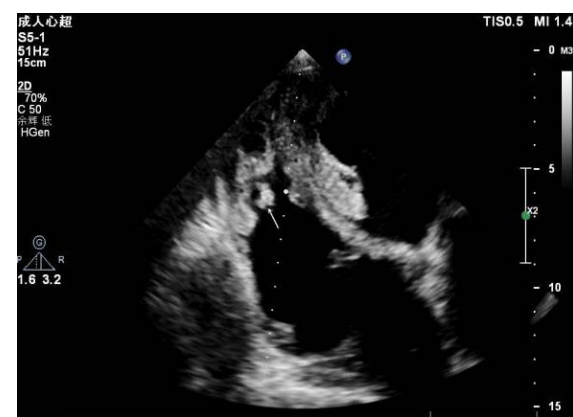

(c)

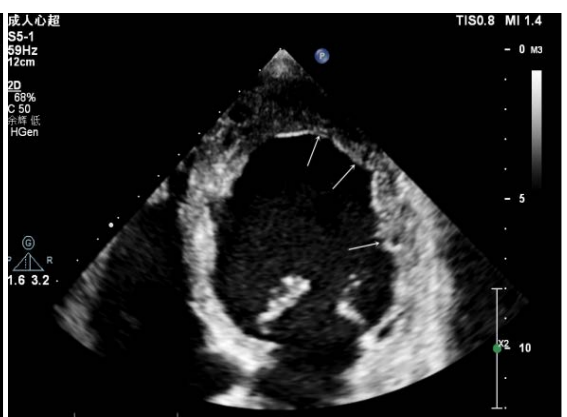

(d)

Figure 1. Transthoracic echocardiography demonstrated massive thrombus formation (arrow) within left ventricular cavity (a), significantly reduced thrombotic masses after two days of anticoagulation therapy in left and right ventricular cavity (b and c), and delayed revealed severe non-compacted myocardium with excessive trabeculae and deep recesses after six days of anticoagulation therapy (d).

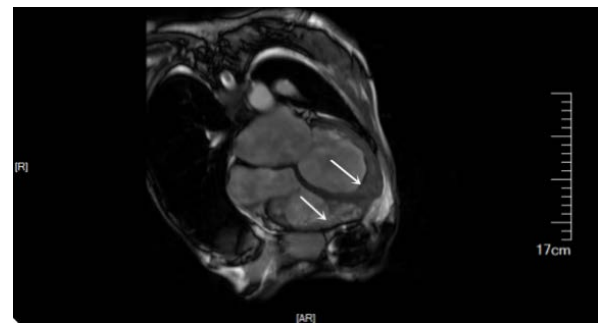

(a)

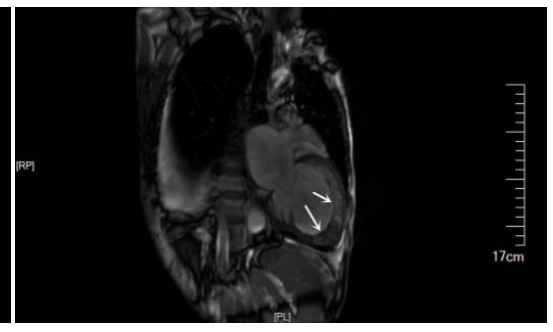

(b)

Figure 2. Cardiac four chamber cine MRI image demonstrated a thick non compact endocardial layer with excessive trabeculae and deep recesses (arrow) in the apical and lateral segments (a); Two chambers view showed a non-compacted hypertrabeculated spongy layer (arrows) was identified, with a ratio greater than 2.3:1 of the compacted layer at the end of diastole (b). 


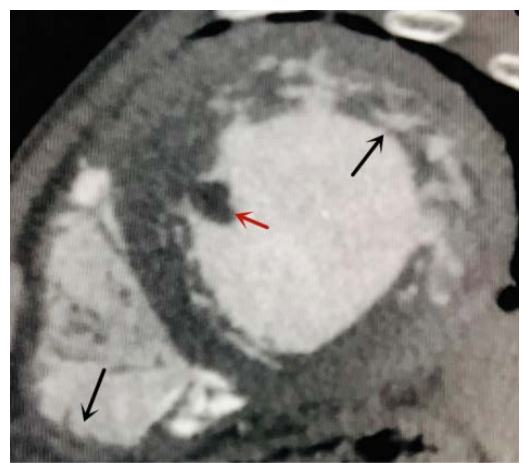

(a)

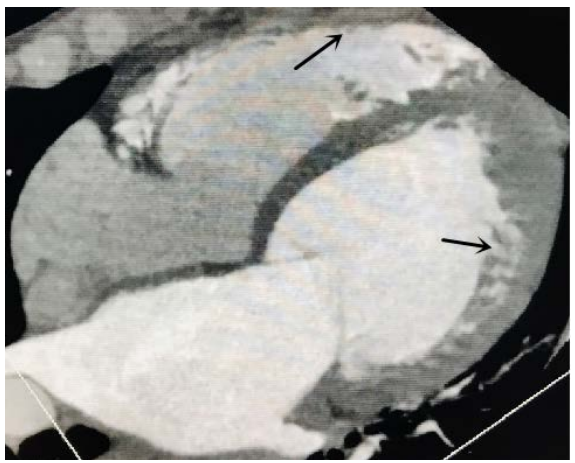

(b)

Figure 3. Retrospective coronary computed tomography angiography showed massive thrombus formation (red arrow) and non-compacted myocardium (black arrows) in left ventricle (a); Numerous prominent trabeculations and deep intertrabecular recesses (arrows) in left and right ventricle (b).

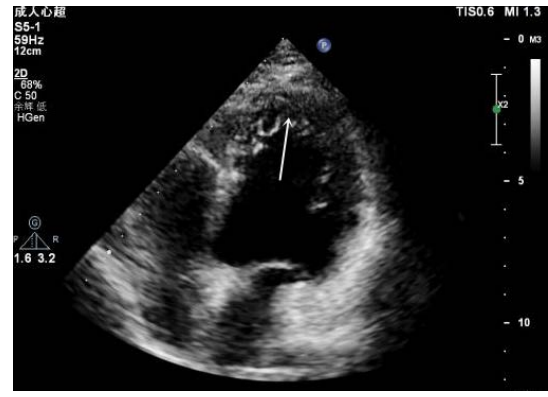

(a)

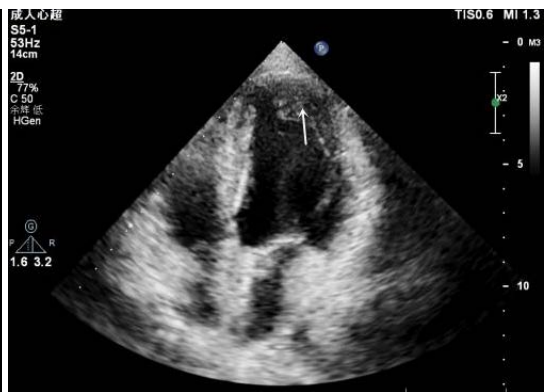

(b)

Figure 4. Echocardiography of the patient's 38- and 31-year-old daughters demonstrated a thickened and reticulated trabecular muscle (arrows) in the apex of left ventricle (a and b).

The patient recovered and was discharged seven days later on the following medications: angiotensin-converting enzyme inhibitor, coenzyme Q10, furosemide, spironolactone, betabloc, and warfarin. Yet, after repeated admitted to the heart failure unit for aggravating heart failure, he passed away four months later.

\section{Discussion}

Non-compaction cardiomyopathy is a rare form of cardiomyopathy characterised by spongy hypertrabeculation of the ventricular myocardium resulting from failure or arrested compaction of the myocardium in utero, with uncertain etiology and mechanism but is generally considered autosomal dominant inheritance. However, it can also be sporadic [6]. Although LVNC mainly affects the left ventricle, isolated right ventricular and biventricular non-compaction may also occur. In the early embryo, the heart is a loose, interwoven mesh of muscle fibers. The developing myocardium gradually condenses, and the large spaces within the trabecular meshwork disappear, resulting in condensing and compaction of the ventricular myocardium and solidification of the endocardial surfaces [7]. These changes mainly occur between 5 and 8 weeks of embryonic life, pro- 
ceeding from the epicardium to endocardium and from the base of the heart to the apex [8]. But recently, it has been proposed that acquired triggers are associated with the formation of non-compacted myocardium. A case report published in 2019 indicated that LVNC could be a myocardial response to acquired triggers, such as drug toxicity, rather than a primary cardiomyopathy [9].

In this perspective, our case raises a relevant hypothesis that the application of paclitaxel and fluorouracil potentially exacerbated deterioration of cardiac intrinsic anomaly structure formation in a patient with non-compaction cardiomyopathy. Therefore, the role of antineoplastic drugs play in progression of non-compaction cardiomyopathy may suggest more considerable treatment protocols applied in those patient, which provides a new idea in future prognostic improvement.

Our patient had heart failure since he received post-operative chemotherapy with paclitaxel and fluorouracil. Fluorouracil-related cardiotoxicity is incompletely understood and may be related to coronary thrombosis, arterial inflammation, spasm or contraction, and direct damage to the myocardium [10]. Cardiac toxic effects from paclitaxel associated with hypotension and myocardial damage, manifesting as grade 3 or 4 left ventricular end systolic diameter (LVSD) or asymptomatic LVEF decline, was low [11]. In addition, fluorouracil and paclitaxel related cardiotoxicity are mostly reversible after drug withdrawal. However, their effect on the trabeculae of the myocardium remains unclear and requires further investigation. In our case, considering the patient received no cardiotoxic drugs or other cardiac risk factors after chemotherapy, his fundamental myocardial disease may be more susceptible to the rapid increase of non-compacted myocardium caused by fluorouracil and paclitaxel related cardiotoxicity. Our patient's non-compacted myocardium occurred in the left and right ventricles, especially in the apex and lateral walls of left ventricle. Combined with the echocardiographic results performed 3 years ago, these facts strongly suggest that antineoplastic drugs may cause the deterioration of cardiac functions of non-compaction cardiomyopathy by promoting trabecular formation in the myocardium. More clinical evidence is required to clarify whether the effect of antineoplastic drugs on the myocardium of patients with non-compaction cardiomyopathy is an accidental case or a common phenomenon.

Traditionally, diagnosis is heavily reliant on advanced imaging with echocardiography or CMR. As the first-line tool for diagnosing non-compaction cardiomyopathy, echocardiography is low-cost, convenient, non-invasive, and has high sensitivity to monitor and evaluate the status of blood flow in the ventricular cavity in real time. The most commonly used echocardiographic standard was proposed by Jenni et al. [12]. Compared with echocardiography, CMR is a more accurate means of characterizing non-compacted myocardial tissue. The diagnostic criterion for CMR is end-diastolic non-compression/compression > ratio 2.3. CMR is not only the most accurate method for diagnosing non-compaction cardiomyopathy, it can also predict the prognosis of these patients, and identify myocardial fibrosis using advanced gadolinium enhancement (LGE) technology [5]. In our 
case, the structure of the non-compaction of the ventricular myocardium did not appear in the initial echocardiography, which may result from not only the presence of a large number of biventricular thrombus affecting the imaging of the biventricular wall, but also from poor image quality and operator inexperience. Similarly, significant reduction of intracardiac thrombi may have ultimately revealed biventricular trabeculae on the subsequent echocardiograph. Therefore, uncertainties in ultrasonic evaluations may lead to delays in non-compaction cardiomyopathy diagnoses. We are attempting to draw lessons from the identification of multitudinous myocardium by imagological examination. Although non-compaction cardiomyopathy has symptoms similar to DCM, we should make full use of imaging to identify cardiac structural and functional changes to differentiate between DCM and non-compaction cardiomyopathy. To explore the type of cardiomyopathy, we usually use echocardiography and computed tomography in medical practice. Notably, visualization of the left ventricular apex and right ventricle can be limited on echocardiography; for example, apical hypertrophic cardiomyopathy may not be identified [13]. Instead, evidence of ventricular dilatation, increased wall thickness, or subclinical myocardial infarction may be observed on multiplanar reformation (MPR) of coronary CTA. In general, hospitals, especially for those not eligible for MRI and echocardiographic results, cannot adapt to clinical demand. For these patients, we may be able to take a more convenient approach-multi-detector CT (MDCT).

There are no specific treatment guidelines for this type of cardiomyopathy, and no evidence that patients with non-compaction cardiomyopathy have different outcomes and should be treated differently from HF patients [14]. Notably, prevention from thromboembolic events is an important management goal for non-compaction cardiomyopathy. However, limited evidence exists to determine the correct course of antithrombotic medication options partly due to the low prevalence LVNC. Anticoagulation is generally recommended for those presenting with ventricular systolic dysfunction, antecedent systemic embolism, cardiac thrombus, and atrial fibrillation [15]. In our case, the patient was treated with warfarin instead of new oral anticoagulants (NOACs) and showed no significant biventricular thrombus during the two follow-ups after discharge. However, there is still insufficient evidence to support the efficacy of the new oral anticoagulant over vitamin $\mathrm{K}$ antagonists in patients with non-compaction cardiomyopathy combined with thrombus. It is obvious that further studies should address a comprehensive anticoagulation strategy for a reduced risk of bleeding, thromboembolic events, and mortality in this patient population.

\section{Limitations}

There are some limitations in our case report. We have not performed genetic testing for patients before and after taking antineoplastic drugs, nor have we investigated the mechanism by which antineoplastic drugs aggravate cardiac functional insufficiency, so we cannot pinpoint the specific role antineoplastic drugs 
played in the process of trabecular formation in patients with non-compaction cardiomyopathy from a pathological perspective. In addition, we failed to do genetic testing for the patient's daughters, so we could not analyze the genotype of non-compaction cardiomyopathy among those family members.

\section{Conclusion}

Our case report presents a patient with primary non-compaction cardiomyopathy showed a rapid deterioration of cardiac function combined with a thin myocardial wall, prominent biventricular trabeculations, and thrombogenesis following treatment with antineoplastic drugs, which indicates that antineoplastic drugs may potentially accelerate the progression of non-compaction of the ventricular myocardium. In addition, we discuss the process of diagnosis and identification of cardiomyopathy and propose multi-detector CT, as an alternative imagological examination, may provide us a new perspective to diagnose non-compaction cardiomyopathy. However, more studies are required to clarify the pathogeny of non-compaction cardiomyopathy.

\section{Ethics Approval and Consent to Participate}

Not applicable.

\section{Consent}

Written informed consent was obtained from the patient for publication of this Case report and any accompanying images. A copy of the written consent is available for review by the Editor of this journal.

\section{Availability of Data and Materials}

Data sharing is not applicable to this article as no datasets were generated or analysed during the current study.

\section{Funding}

This study was supported by the Nature Science Foundation of China (grant No. 81873513, 81600574, and 30871042), Key Projects of Shaanxi Science and Technology Research and Development Plan (No. 2018ZDXM-SF-049).

\section{Authors' Contributions}

Dr. Gang Tian: diagnosed the case and instructed the patient's treatment. Mr. Chaodi Luo and Dr. Yanjie Yang: collected the primary data and drafted the initial manuscript. Dr. Chun Yang: Helped process echocardiographic images. Dr. Xiang Hao and Ms. Zhenzhen Duan: assisted with the treatment of the patient. Dr. Guoliang Li: Assisted with the analyzation of the case characteristics and gave writing suggestions. All authors contributed to discussions and critically appraised the manuscript. All authors approved the final manuscript as submitted and agree to be accountable for all aspects of the work. 


\section{Acknowledgements}

We thank Editage (http://www.editage.com/) for English language editing.

\section{Conflicts of Interest}

The authors declare that they have no competing interests.

\section{References}

[1] Maron, B.J., Towbin, J.A., Thiene, G., et al. (2006) Contemporary Definitions and Classification of the Cardiomyopathies: An American Heart Association Scientific Statement from the Council on Clinical Cardiology, Heart Failure and Transplantation Committee; Quality of Care and Outcomes Research and Functional Genomics and Translational Biology Interdisciplinary Working Groups; and Council on Epidemiology and Prevention. Circulation, 113, 1807-1816. https://doi.org/10.1161/CIRCULATIONAHA.106.174287

[2] Finsterer, J., Stöllberger, C. and Towbin, J.A. (2017) Left Ventricular Noncompaction Cardiomyopathy: Cardiac, Neuromuscular, and Genetic Factors. Nature Reviews Cardiology, 14, 224-237. https://doi.org/10.1038/nrcardio.2016.207

[3] Chin, T.K., Perloff, J.K., Williams, R.G., et al. (1990) Isolated Noncompaction of Left Ventricular Myocardium. A Study of Eight Cases. Circulation, 82, 507-513. https://doi.org/10.1161/01.CIR.82.2.507

[4] Ikeda, U., Minamisawa, M. and Koyama, J. (2015) Isolated Left Ventricular Non-Compaction Cardiomyopathy in Adults. Journal of Cardiology, 65, 91-97. https://doi.org/10.1016/j.jjcc.2014.10.005

[5] Taghavi, M., Ghaemian, A., Nabati, M., Farsavian, A. and Shokri, M. (2019) The Coexistence of Left Ventricular Non-Compaction Cardiomyopathy, Significant Coronary Artery Disease and Massive Thrombus Formation in Left Ventricular Cavity: A Rare Case Report. Journal of Clinical Ultrasound, 47, 107-110.

https://doi.org/10.1002/jcu.22659

[6] Towbin, J.A. and Jefferies, J.L. (2017) Cardiomyopathies Due to Left Ventricular Noncompaction, Mitochondrial and Storage Diseases, and Inborn Errors of Metabolism. Circulation Research, 121, 838-854. https://doi.org/10.1161/CIRCRESAHA.117.310987

[7] Towbin, J.A., Lorts, A. and Jefferies, J.L. (2015) Left Ventricular Non-Compaction Cardiomyopathy. The Lancet, 386, 813-825. https://doi.org/10.1016/S0140-6736(14)61282-4

[8] Oechslin, E. and Jenni, R. (2011) Left Ventricular Non-Compaction Revisited: A Distinct Phenotype with Genetic Heterogeneity? European Heart Journal, 32, 1446-1456. https://doi.org/10.1093/eurheartj/ehq508

[9] Loria, V., Colizzi, C., Vaccarella, M., Franceschi, F. and Aspromonte, N. (2019) Left Ventricular Noncompaction: Cause or Consequence of Myocardial Disease? A Case Report and Literature Review. Cardiology, 143, 100-104. https://doi.org/10.1159/000500904

[10] Sara, J.D., Kaur, J., Khodadadi, R., et al. (2018) 5-Fluorouracil and Cardiotoxicity: A Review. Therapeutic Advances in Medical Oncology.

https://doi.org/10.1177/1758835918780140

[11] Dang, C., Guo, H., Najita, J., et al. (2016) Cardiac Outcomes of Patients Receiving Adjuvant Weekly Paclitaxel and Trastuzumab for Node-Negative, ERBB2-Positive Breast Cancer. JAMA Oncology, 2, 29-36. 
https://doi.org/10.1001/jamaoncol.2015.3709

[12] Jenni, R., Oechslin, E., Schneider, J., Attenhofer Jost, C. and Kaufmann, P.A. (2001) Echocardiographic and Pathoanatomical Characteristics of Isolated Left Ventricular Non-Compaction: A Step towards Classification as a Distinct Cardiomyopathy. Heart, 86, 666-671. https://doi.org/10.1136/heart.86.6.666

[13] Baksi, A.J. and Pennell, D.J. (2015) Cardiomyopathy. In: Advanced Cardiac Imaging, Woodhead Publishing, Cambridge, 399-438. https://doi.org/10.1016/B978-1-78242-282-2.00013-5

[14] Rooms, I., Dujardin, K. and De Sutter, J. (2015) Non-Compaction Cardiomyopathy: A Genetically and Clinically Heterogeneous Disorder. Presentation of Two Cases and Review of the Literature. Acta Cardiologica, 70, 625-631. https://doi.org/10.1080/AC.70.6.3120173

[15] De Melo, M.D.T., de Araújo Filho, J.B., Parga Filho, J.R., et al. (2015) Noncompaction Cardiomyopathy: A Substrate for a Thromboembolic Event. BMC Cardiovascular Disorders, 15, 7. https://doi.org/10.1186/1471-2261-15-7

\section{List of Abbreviations}

CMR Cardiac magnetic resonance imaging

CT Computed tomography

CTA Computed tomographic angiography

DCM Dilated cardiomyopathy

HF Heart failure

INR International normalized ratio

LGE Gadolinium enhancement

LV Left ventricle

LVEF Left ventricular ejection fraction

LVNC Left ventricular non-compaction

LVSD Left ventricular end systolic diameter

MDCT Multi-detector computed tomography

MPR Multiplanar reformation

NOACs New oral anticoagulants 